\title{
A relação entre o uso de álcool e acidentes de trânsito em Caxias - MA
}

\author{
The relationship between alcohol use and traffic accidents in Caxias - MA \\ La relación entre el consumo de alcohol y los accidentes de tráfico en Caxias - MA
}

Pedro Henrique Medeiros de Andrade ORCID: https://orcid.org/0000-0003-1819-9540 Centro Universitário de Ciências e Tecnologia do Maranhão, Brasil E-mail: pedro.medeiroscx@gmail.com

Kaio Germano Sousa da Silva ORCID: https://orcid.org/0000-0003-4236-6230 Faculdade do Vale Elvira Dayrell, Brasil E-mail: kaiogsds@hotmail.com

Luana Pereira Ibiapina Coêlho ORCID: https://orcid.org/0000-0002-2054-959X Faculdade de Venda Nova do Imigrante, Brasil E-mail: luana_ibiapina@hotmail.com

Vinícius Sampaio Melo do Nascimento ORCID: https://orcid.org/0000-0001-6651-6112 Centro Universitário de Ciências e Tecnologia do Maranhão, Brasil E-mail: vinirocksk8@gmail.com Danielle Carvalho Rocha

ORCID: https://orcid.org/0000-0002-4022-037X

Universidade Estadual do Maranhão, Brasil E-mail: daniellerocha.slz@gmail.com

Lucas José Miranda Gomes

ORCID: https://orcid.org/0000-0002-1707-516X Centro Universitário de Ciências e Tecnologia do Maranhão, Brasil E-mail: lucas40jose@gmail.com

Mariana Rodrigues Silva

ORCID: https://orcid.org/0000-0002-9072-9174 Centro Universitário de Ciências e Tecnologia do Maranhão, Brasil E-mail: rodriguessmaari@gmail.com

George do Carmo Leão

ORCID: https://orcid.org/0000-0001-9106-1500 Centro Universitário de Ciências e Tecnologia do Maranhão, Brasil E-mail: georgeleao13@gmail.com

Karolayne Brito Silva ORCID: https://orcid.org/0000-0002-3679-9091 Centro Universitário de Ciências e Tecnologia do Maranhão, Brasil E-mail: karolayneb092@gmail.com

Francisca Jorlanna da Silva Rocha

ORCID: https://orcid.org/0000-0003-2942-6585

Centro Universitário de Ciências e Tecnologia do Maranhão, Brasil E-mail: silvajorlanna04@gmail.com

Atalia Talita da Silva

ORCID: https://orcid.org/0000-0003-3726-685X Centro Universitário de Ciências e Tecnologia do Maranhão, Brasil E-mail: talitaatalia877@gmail.com

Washington Walber Macedo dos Santos ORCID: https://orcid.org/0000-0003-4577-1143 Centro Universitário de Ciências e Tecnologia do Maranhão, Brasil E-mail: mwashingtonwalber@gmail.com

Isadora Sayonara Ferreira Coelho ORCID: https://orcid.org/0000-0002-3300-6244 Centro Universitário de Ciências e Tecnologia do Maranhão, Brasil E-mail: isaaebella3006@gmail.com

Maria de Fátima Silva

ORCID: https://orcid.org/0000-0002-4422-6947

Centro Universitário de Ciências e Tecnologia do Maranhão, Brasil

Maria Eduarda Leal de Carvalho Santos

ORCID: https://orcid.org/0000-0003-3400-0570 Centro Universitário de Ciências e Tecnologia do Maranhão, Brasil
E-mail: eduardalealcs@ gmail.com 


\author{
Amós da Silva Uchôa \\ ORCID: https://orcid.org/0000-0001-7313-098X \\ Centro Universitário de Ciências e Tecnologia do Maranhão, Brasil \\ E-mail: amosuchoa@hotmail.com \\ Rondinelle dos Santos Chaves \\ ORCID: https://orcid.org/0000-0003-4941-8005 \\ Instituo Sírio-Libanês de Ensino e Pesquisa, Brasil \\ E-mail: rondinelledsch@hotmail.com \\ Letícia Aparecida Santos Silva \\ ORCID: https://orcid.org/0000-0002-8000-7071 \\ Centro Universitário de Ciências e Tecnologia do Maranhão, Brasil \\ E-mail: leticia.odonto01@outlook.com
}

\begin{abstract}
Resumo
O Brasil apresenta altas taxas de mortalidade decorrente de acidentes de trânsito, e em sua grande maioria, proveniente de colisões motociclistas. Buscando assim entender qual o impacto do uso de álcool nos acidentes de trânsito, o presente estudo objetivou caracterizar a prevalência de acidentes de trânsito causados pelo consumo de álcool, bem como a realização de um levantamento dos índices de acidentes de trânsito entre pessoas com níveis de idades variados. Trata-se de um estudo descritivo, documental, com abordagem quantitativa realizado em um Hospital do município de Caxias-MA, com as fichas de pacientes com de diferentes idades, que tenham sofrido acidentes de trânsito entre as datas de $1^{\circ}$ de Agosto de 2019 e 31 de Dezembro de 2019. Como resultados se ratificam que foram analisados 457 prontuários de acidentes de trânsito, dentre eles, 165 pertencem a pacientes com idades entre 12 e 20 anos, representando assim $36 \%$ no total dos dados presentes, nas quais 93 pessoas fizeram o uso de álcool, uma amostra percentual de 53,3\% da totalidade, 4,2\% no mês de agosto, 9,7\% no mês de setembro, 13,9\% no mês de outubro, $7,8 \%$ no mês de novembro e $20,6 \%$ no mês de dezembro. Pode-se concluir que o uso de álcool aumenta consideravelmente o índice de acidentes de trânsito, tendo como principal vítimas os adolescentes, em vista de alguns fatores como: inexperiência, a falta de habilitação e a imprudência.
\end{abstract}

Palavras-chave: Acidentes de trânsito; Adolescente; Consumo de bebidas alcoólicas.

\begin{abstract}
Brazil has high mortality rates resulting from traffic accidents, and the vast majority from motorcycle collisions. In order to understand the impact of alcohol use on traffic accidents, this study aimed to characterize the prevalence of traffic accidents caused by alcohol consumption, as well as to carry out a survey of traffic accident rates among people with levels of varied ages. This is a descriptive, documentary study with a quantitative approach carried out in a Hospital in the city of Caxias-MA, with the files of patients of different ages, who have suffered traffic accidents between the dates of August 1, 2019 and December 31, 2019. The results confirm that 457 records of traffic accidents were analyzed, among them, 165 belong to patients aged between 12 and 20 years, thus representing $36 \%$ of the total data present, in which 93 people did alcohol use, a percentage sample of 53.3\% of the total, $4.2 \%$ in August, $9.7 \%$ in September, $13.9 \%$ in October, $7.8 \%$ in the month of November and $20.6 \%$ in December. It can be concluded that the use of alcohol considerably increases the rate of traffic accidents, with adolescents as the main victims, in view of some factors such as: inexperience, lack of license and recklessness.
\end{abstract}

Keywords: Accidents, traffic; Adolescent; Alcohol drinking.

\title{
Resumen
}

Brasil tiene altas tasas de mortalidad por accidentes de tráfico y la gran mayoría por colisiones de motocicletas. Con el fin de comprender el impacto del consumo de alcohol en los accidentes de tráfico, este estudio tuvo como objetivo caracterizar la prevalencia de accidentes de tráfico provocados por el consumo de alcohol, así como realizar una encuesta de tasas de accidentes de tráfico entre personas con niveles de distintas edades. Se trata de un estudio descriptivo, documental con abordaje cuantitativo realizado en un Hospital de la ciudad de Caxias-MA, con los expedientes de pacientes de diferentes edades, que han sufrido accidentes de tránsito entre las fechas del 1 de agosto de 2019 y el 31 de diciembre. 2019. Los resultados confirman que se analizaron 457 registros de accidentes de tránsito, entre ellos, 165 pertenecen a pacientes con edades entre 12 y 20 años, lo que representa el 36\% del total de datos presentes, en los que 93 personas hicieron el consumo de alcohol, un porcentaje muestra del 53,3\% del total, $4,2 \%$ en agosto, $9,7 \%$ en septiembre, $13,9 \%$ en octubre, $7,8 \%$ en el mes de noviembre y $20,6 \%$ en diciembre. Se puede concluir que el consumo de alcohol aumenta considerablemente la tasa de accidentes de tránsito, siendo los adolescentes las principales víctimas, ante algunos factores como: inexperiencia, falta de licencia y temeridad.

Palabras clave: Accidentes de tránsito; Adolescente; Consumo de bebidas alcohólicas.

\section{Introdução}

O Brasil apresenta altas taxas de mortalidade decorrente de acidentes de trânsito, e em sua grande maioria, proveniente de colisões motociclistas. Diversos fatores de risco interagem para a ocorrência dos mesmos, como: velocidade (se 
tornando inapropriada ou excessiva), o uso de álcool e outras drogas que atuam como coadjuvantes, a presença de um personagem vulnerável (como crianças e idosos), más condições da estrada, além de alterações psicológicas que podem interferir diretamente na atuação (como pensamentos suicidas, sintomatologia depressiva, atos impulsivos relacionados a comportamentos violentos e destrutivos). Tem-se observado também que uma grande quantidade de acidentes de trânsito é constituída de adolescente que atuam de forma irregular provocando tais fatalidades (Davoren, Demant, Shiely, \& Perry, 2016; Ponce et al., 2019; Robison, Tarzi, Zhou, \& Bailey, 2020).

A presente pesquisa tem a pretensão de desenvolver um estudo sobre a relação entre o uso de álcool e acidentes de trânsito em Caxias, município do estado do Maranhão. Neste sentido, entende-se que o consumo de álcool reduz o reflexo corporal aos estímulos externos. Além disso, grande parte dos acidentes de trânsito ocorre entre jovens e adolescentes que não possuem habilitação e realizam a ingestão de bebidas alcoólicas (Lopes et al., 2012).

Um estudo de Melo (2018) e Smailovic, Lipovac, Pešić e Antić (2019) recentemente pode-se observar um aumento significativo no número de motocicletas e condutores de veículos, bem como uma tendência decrescente na categoria de pedestres e um aumento da mortalidade em todos os anos e em todas as categorias, especialmente para motocicletas e ocupantes de automóveis. O aumento da taxa de mortalidade por acidentes de trânsito consiste em um fenômeno mundial na última década, principalmente em países de baixa e média renda.

A lei no 9.503, de 23/09/97 que estabelece o Código de Trânsito Brasileiro (CTB), conceitua trânsito como o uso das vias terrestres por veículos, pessoas e animais, em grupos ou isolados para fins de circulação, parada, estacionamento e operação de carga ou descarga. Complementando, o Departamento de Informática do Sistema Universal de Saúde (DATASUS) utiliza o conceito da Classificação Internacional de Doenças e Problemas Relacionados à Saúde para conceituar e classificar os acidentes de transporte (Lei n ${ }^{\circ}$ 9.503, 1997).

A escolha dessa temática se deu pelo fato de que o consumo de bebidas alcoólicas aumenta consideravelmente as chances de um acidente de trânsito seja ele por meio de uma colisão, atropelamento ou queda, no qual o consumo de álcool se caracteriza como responsável pela redução da velocidade de reação aos estímulos externos, além de que o fator experiência no trânsito se torna crucial para o desenvolvimento de um acidente. Cabe ainda ressaltar que esse estudo é relevante para os mais diversos profissionais da saúde, bem como para a própria população, tornando-se assim possível ampliar seus conhecimentos mediante os mais diversos casos de adolescentes que acabam se envolvendo em um acidente de trânsito após o uso irregular de bebidas alcoólicas.

O interesse de realizar esta pesquisa sobre a relação do uso de álcool e acidentes de trânsito no município de Caxias MA, deu-se também pelo fato de trabalharmos num hospital da cidade e termos percebido que este recebe continuamente casos de vítimas de traumas automobilísticos, provenientes de acidentes, colisões e atropelamentos, índice esse que se apresenta em níveis elevados, no entanto em maior quantidade em pessoas com faixa etária de doze até vinte anos, e em grande maioria, demonstrando comportamento alterado devido ao uso de bebidas alcoólicas.

Diante dessa realidade, este estudo tem como problemática: "Qual o impacto do uso de álcool nos acidentes de trânsito?”. Desse modo, a pesquisa possui como objetivo a caracterização da prevalência de acidentes de trânsito causados pelo consumo de álcool, bem como a realização de um levantamento dos índices de acidentes de trânsito entre pessoas com níveis de idades variados.

\section{Metodologia}

\section{Tipo de pesquisa}

Trata-se de um estudo descritivo, documental, de abordagem quantitativa. O estudo descritivo tem como por intenção analisar, registrar e fazer um julgamento dos fenômenos observados, de forma que nesse tipo de estudo o pesquisador não pode 
vir a interferir na ocorrência e resolução dos fatos, que foram observados (Barros \& Lehfeld, 2007; Potter \& Perry, 2013).

A pesquisa documental compreende o método que parte de um ponto no tempo em que a pesquisa foi realizada, onde alguns eventos e processos foram analisados respectivamente, ou seja, de um ponto no presente para o passado, com respeito ao seu significado para as histórias de vida individuais ou coletivas, além de envolver a definição de grupos apropriados para comparação, justificando os limites do tempo a serem investigados, checando a questão da pesquisa e decidindo que fontes e documentos devem ser usados (Dyniewicz, 2009).

A pesquisa quantitativa é aquela em que se coleta e analisa dados quantitativos sobre variáveis. Assim é possível identificar a natureza profunda das realidades, seu sistema de relações, e sua estrutura dinâmica (Flick, 2013).

\section{Local, período de coleta e amostra}

A pesquisa foi realizada em um Hospital que fica localizado no município de Caxias, que está situada na região leste do estado do Maranhão a 374 km da capital maranhense, São Luís e 76 km da capital do Piaúí, Teresina, com área territorial em 2015 de $5.196,771 \mathrm{~km}^{2}$.

De acordo com o hospital em questão, ele apresenta a capacidade de atender os mais diversos pacientes do município e abrange a região (Aldeias Altas, Coelho Neto, São João do Sóter, Buriti entre outros), ressaltando que a unidade realiza aproximadamente 300 atendimentos por dia. Foram parte do estudo as fichas dos pacientes atendidos entre o período do dia 1 de agosto de 2019 a 31 de dezembro de 2019.

\section{Critérios de inclusão e exclusão}

Os critérios de inclusão foram: pacientes que tenham sofrido acidentes de trânsito entre as datas de $1^{\circ}$ de agosto de 2019 e 31 de dezembro de 2019. Os critérios de exclusão foram: pacientes que não foram atendidos no Hospital escolhido para realização da pesquisa, fichas rasuradas ou que impossibilitem sua compreensão.

\section{Métodos de coleta e análise dos dados}

Para a obtenção dos dados, foram utilizados questionários, prontuários, fichas ambulatoriais, além da ficha de atendimento Hospital, que contém dados como idade, sexo, cor da pele referida, procedência, horário de chegada na unidade hospitalar, dia da semana e mês de atendimento, queixa principal de acordo com o sistema orgânico comprometido, sinais e sintomas apresentados, antecedentes pessoais e hábitos de vida, cor da classificação de risco ao final da avaliação realizada pelo enfermeiro, setor de atendimento, perfil do usuário, circunstâncias de atendimento, demanda inadequada, hipótese diagnóstica e exames solicitados.

Os dados foram coletados (atendimentos realizados conforme cada mês), depois estratificados e em seguida analisados conforme CID (Classificação Internacional de Doenças) de cada usuário, e depois foi realizado cálculo amostral para cada mês. Os dados foram analisados em planilhas do programa Microsoft Office Excel for Windows, por meio de estatística básica.

\section{Aspectos éticos}

A pesquisa acatou todos os aspectos éticos que envolvem os estudos dessa natureza, conforme o deliberado pelo Conselho Nacional de Saúde, com o devido respaldo da resolução 466/2012. O presente estudo foi submetido à Plataforma Brasil e ao Comitê de Ética e Pesquisa (CEP) do Centro Universitário de Ciências e Tecnologia do Maranhão - UNIFACEMA, parecer: 3.896.266, aprovado com o CAAE: $\mathrm{n}^{\circ}$ 28300620.2.0000.8007. Conforme a resolução de $\mathrm{n}^{\circ} 466 / 12$, que tem o objetivo de garantir os direitos deveres dos participantes da pesquisa (Portaria ${ }^{\circ} 466,2012$ ). 


\section{Resultados e Discussão}

Conforme o resultado geral da pesquisa, foram analisados 457 prontuários hospitalares e fichas ambulatoriais de pacientes que sofreram acidentes de trânsito, entre os meses de agosto a dezembro de 2019. Nos dados expostos na Tabela 1, pode-se observar que foram analisadas ao total de 36 fichas ambulatoriais no mês de agosto.

Tabela 1. Demonstrativo dos acidentes de trânsito relacionados ao mês de agosto de 2019, (N=36). Caxias-MA.

\begin{tabular}{ccc}
\hline $\begin{array}{c}\text { Variável } \\
\text { Idade }\end{array}$ & $\begin{array}{c}\text { Frequência } \\
\text { Relativa }\end{array}$ & $\%$ \\
\hline $12-20$ & 21 & 58,3 \\
$21-30$ & 4 & 11,1 \\
$31-40$ & 3 & 8,4 \\
$41-50$ & 6 & 16,6 \\
$51-60$ & 2 & 5,6 \\
$<61$ & 0 & 0 \\
\hline
\end{tabular}

Fonte: Pesquisa de Campo (2020).

Na Tabela 2, correspondente ao mês de setembro, é visível o aumento na quantidade de fichas ambulatoriais analisadas, pois se estabelece como finalizado o setor de pronto atendimento hospitalar. Conforme o expositivo analisou-se 88 fichas.

Tabela 2. Demonstrativo dos acidentes de trânsito, relacionado ao mês de setembro de 2019, (N=88). Caxias-MA.

\begin{tabular}{ccc}
\hline $\begin{array}{c}\text { Variável } \\
\text { Idade }\end{array}$ & $\begin{array}{c}\text { Frequência } \\
\text { Relativa }\end{array}$ & $\%$ \\
\hline $12-20$ & 38 & 43,2 \\
$21-30$ & 17 & 19,4 \\
$31-40$ & 14 & 15,9 \\
$41-50$ & 9 & 10,2 \\
$51-60$ & 9 & 10,2 \\
$<61$ & 1 & 1,1 \\
\hline
\end{tabular}

Fonte: Pesquisa de Campo (2020).

A Tabela 3 relaciona a quantidade de fichas ambulatoriais com registro nos quais constam 101 acidentes de trânsito do mês de outubro, com variáveis de gênero e idade, expõe-se que esse resultado está relacionado à análise de todas as fichas.

Tabela 3. Demonstrativo dos acidentes de trânsito, relacionado ao mês de outubro de 2019, (N=101). Caxias-MA.

\begin{tabular}{ccc}
\hline $\begin{array}{c}\text { Variável } \\
\text { Idade }\end{array}$ & Frequência Relativa & $\%$ \\
\hline $12-20$ & 33 & 32,7 \\
$21-30$ & 28 & 27,8 \\
$31-40$ & 21 & 20,8 \\
$41-50$ & 12 & 11,9 \\
$51-60$ & 5 & 4,9 \\
$<61$ & 2 & 1,9 \\
\hline
\end{tabular}

Fonte: Pesquisa de Campo (2020).

$\mathrm{Na}$ Tabela 4, pode-se observar que foram analisadas ao total 112 fichas ambulatoriais correspondentes ao número de acidentes de trânsito ocorridos no mês de novembro de 2019. 
Tabela 4. Demonstrativo dos acidentes de trânsito, relacionado ao mês de novembro de 2019, (N=112). Caxias-MA.

\begin{tabular}{ccc}
\hline $\begin{array}{c}\text { Variável } \\
\text { Idade }\end{array}$ & Frequência Relativa & $\%$ \\
\hline $12-20$ & 26 & 23,3 \\
$21-30$ & 47 & 41,9 \\
$31-40$ & 21 & 18,8 \\
$41-50$ & 11 & 9,8 \\
$51-60$ & 4 & 3,6 \\
$<61$ & 3 & 2,6 \\
\hline
\end{tabular}

Fonte: Pesquisa de Campo (2020).

Na Tabela 5, foram observadas 120 fichas ambulatoriais, visto que as pessoas com idades entre 12 e 20 anos apresentaram um percentual de 39,1\% em relação ao total de acidentes acorridos no mês de dezembro de 2019.

Tabela 5. Demonstrativo dos acidentes de trânsito, relacionado ao mês de dezembro de 2019, (N=120). Caxias-MA.

\begin{tabular}{|c|c|c|}
\hline $\begin{array}{c}\text { Variável } \\
\text { Idade }\end{array}$ & Frequência Relativa & $\%$ \\
\hline $12-20$ & 47 & 39,1 \\
\hline $21-30$ & 33 & 27,5 \\
\hline $31-40$ & 11 & 9,1 \\
\hline $41-50$ & 9 & 7,5 \\
\hline $51-60$ & 13 & 11,8 \\
\hline$<61$ & 6 & 5 \\
\hline
\end{tabular}

Fonte: Pesquisa de Campo (2020).

No Gráfico 1, têm-se uma representação geral dos dados analisados, a partir do mês de agosto até dezembro de 2019 , de forma que fez-se uma somatória total e sua divisão perante a faixa etária amostral, como pode-se observar, foram coletados 165 fichas ambulatoriais, com idades entre 12 e 20 anos, representando assim $36 \%$ no total dos dados presentes. A próxima série de idades analisadas foi de 21 a 30 anos, tendo também grande representatividade, com 129 fichas ambulatoriais no total, portanto, apresentando um número amostral de 28\%, já a faixa etária de 31 até os 40 anos mostrou um total de 70 fichas analisadas, constituindo $16 \%$ de todo o cálculo amostral, as idades de 41 até os 50 anos continuaram decrescendo, em vista que o número de fichas ambulatoriais e prontuários examinados foram de apenas 47, fornecendo assim apenas $10 \%$ de sua totalidade e com apenas 33 fichas observadas e constituindo $7 \%$ do total das pesquisas ficaram as pessoas com idade entre 51 e 60 anos, e aquelas acima de 61 anos representaram apenas 3\% do total de acidentes, com 12 fichas avaliadas. 
Gráfico 1. Demonstrativo do número de acidentes por faixa etária do período de agosto a dezembro de 2019. (N=456). CaxiasMA.

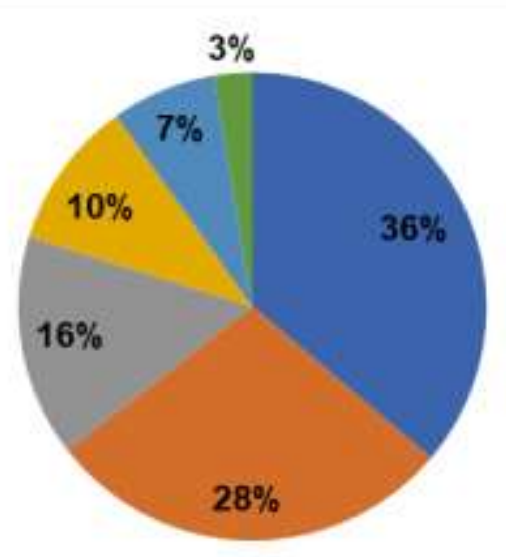

$=12-20$ ANOS $=21-30$ ANOS

$=31-40$ ANOS $=41-50$ ANOS

$=51-60$ ANOS $=<60$ ANOS

Fonte: Pesquisa de Campo (2020).

Nesse enfoque, Andrade e Jorge (2016) e Rocha, Macacare, Abrahao e Ekuni (2019) demonstram que em suas análises, as vítimas dos acidentes de trânsito ocorrem com mais frequência em jovens e adultos, no qual essa situação pode ser explicada pelo consumo abusivo de bebidas alcoólicas, após isso, se expõem ao risco de dirigir.

Muitas vezes o consumo de bebida alcoólica por adolescentes é influenciado pelo próprio ambiente familiar, pois ele estimula o desenvolvimento da conduta e da personalidade. Além disso, são influenciados também pelo grupo de amigos, por sentirem a necessidade de serem aceitos (Benincasa et al., 2018; Ferreira, Veloso, Coutinho, Lira, \& Lira, 2019).

Na Tabela 6, apresenta-se o expositivo que relaciona o número de pessoas com faixa etária de 12 a 20 que sofreram algum acidente de trânsito, com o número de adolescentes que fizeram o uso de bebidas alcoólicas, em um período compreendido entre agosto e dezembro de 2019, de forma que, é possível observar que foram examinadas um total de 165 fichas ambulatoriais, nas quais 93 abrangem pessoas que fizeram o uso de álcool, mostrando assim uma amostra percentual de $53,3 \%$ da totalidade. Nos meses analisados, dezembro possui sua maior quantidade com 47 acidentes de trânsito, destes, 34 são de adolescentes que consumiram álcool, mostrando uma taxa de 20,6\% do total analisado.

Tabela 6. Demonstração do número de adolescentes que consumiram álcool e se envolveram em acidentes de trânsito no período de agosto a dezembro de 2019. ( $\mathrm{N}=165)$. Caxias - MA.

\begin{tabular}{lccc}
\hline & $\begin{array}{c}\mathbf{N}^{\circ} \text { de Acidentes de } \\
\text { Trânsito }\end{array}$ & $\begin{array}{c}\mathbf{N}^{\circ} \text { de adolescentes que } \\
\text { Relataram uso de álcool }\end{array}$ & $\%$ \\
\hline Agosto & 21 & 7 & 4,2 \\
Setembro & 38 & 16 & 9,7 \\
Outubro & 33 & 23 & 13,9 \\
Novembro & 26 & 13 & 7,8 \\
Dezembro & 47 & 34 & 20,6 \\
\hline Total & 165 & 93 & 53,3 \\
\hline
\end{tabular}

Fonte: Pesquisa de Campo (2020).

O uso do álcool com a ocorrência de acidentes e violências deixa claro que o seu uso favorece a inclusão do indivíduo em comportamentos alterado, além do que, referem que jovens que abusam de bebida alcoólica apresentam até três vezes mais 
chance de desenvolverem algum acidente motociclístico, quando comparados aos que não fazem o consumo dessa substância (Soares, Pereira, Moraes, \& Vianna, 2012; Santos, 2014).

Os Gráficos 2 e 3 constituem um comparativo, de forma que eles apresentam a disposição dos dados obtidos entre o mês de agosto a dezembro de 2019, no entanto, expressando em números a quantidade de homens e mulheres com faixa etária de 12 a 20 anos, acidentados entre o período pesquisado, comparando assim com o número de pessoas com a mesma faixa etária, que tenham realizado o uso de bebidas alcoólicas.

O mês de agosto teve apenas 21 acidentes de trânsito envolvendo pessoas com idade entre 12 e 20 anos, dentre eles 16 homens ( 6 fizeram uso de bebidas alcoólicas) e 5 mulheres (apenas 1 relata o uso de álcool), o mês de setembro teve um aumento, com 38 acidentes, sendo 31 homens (um total de 12 homens relatam o uso de bebidas) e 7 mulheres (um total de 4 confirmaram o uso de álcool), o mês de outubro verifica um total de 33 acidentes, com 22 homens (sendo que 18 ingeriram álcool) e 11 mulheres (apenas 5 descreveram o uso de bebidas alcoólicas), no mês de novembro o número total de acidentes envolvendo adolescentes foi de 26, composto por 15 homens (10 referem o uso de álcool) e 11 mulheres (apenas 3 referem o uso do álcool), em dezembro, o último mês estudado, apresentou-se um aumento perante o número de acidentados, 47 no total, com prevalência de homens, com 39 indivíduos ( 28 constatam o uso do álcool) e apenas 8 mulheres (sendo que 6 relataram o uso de álcool).

Os adolescentes estão mais expostos, por conta que são movidos pela curiosidade e as sensações de prazer, de forma que, apresentam-se com maior evidência no sexo masculino, assim como a predominância no trânsito, explicado pelos mais diversos fatores, sejam sociais, culturais e econômicos (Andrade \& Jorge, 2016; Moise, 2020). No Brasil, o risco de acidentes com veículos associados ao uso de álcool pode ser considerado um importante problema de saúde pública, com grande impacto na morbimortalidade (Oliveira et al., 2020).

Gráfico 2. Expositivo do número de acidentes total do mês, com divisão de gênero.

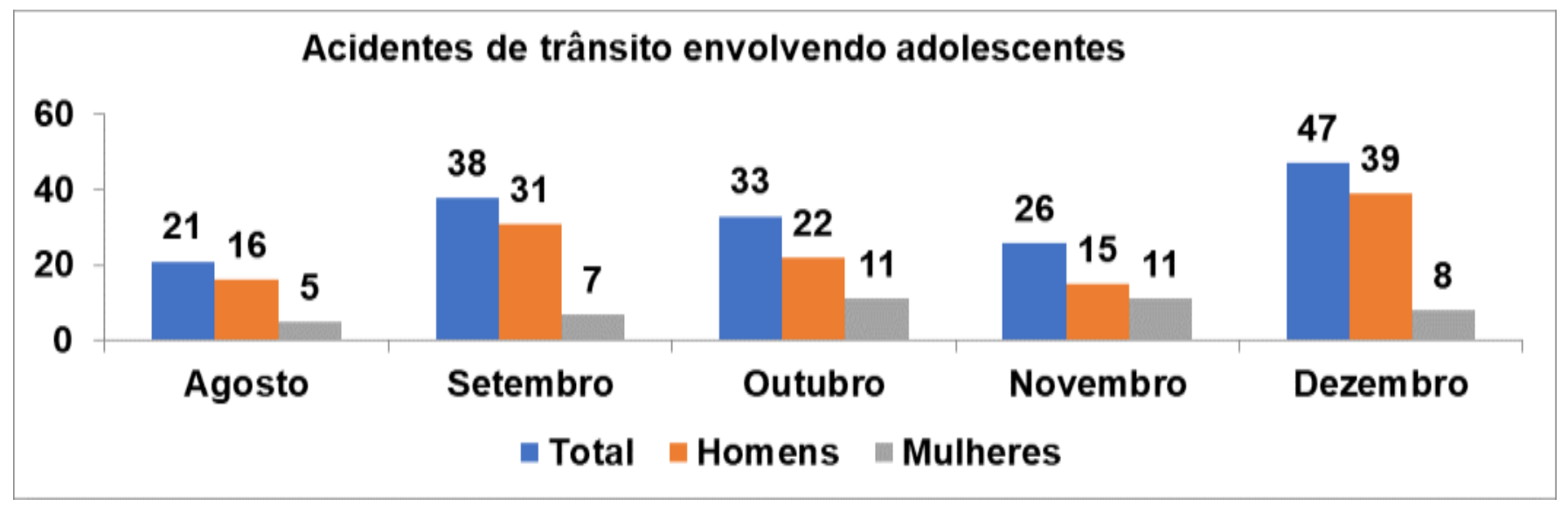

Fonte: Pesquisa de Campo (2020). 
Gráfico 3. Expositivo do número de acidentes total do mês, entre adolescentes que realizaram o consumo de álcool, com divisão de gênero.

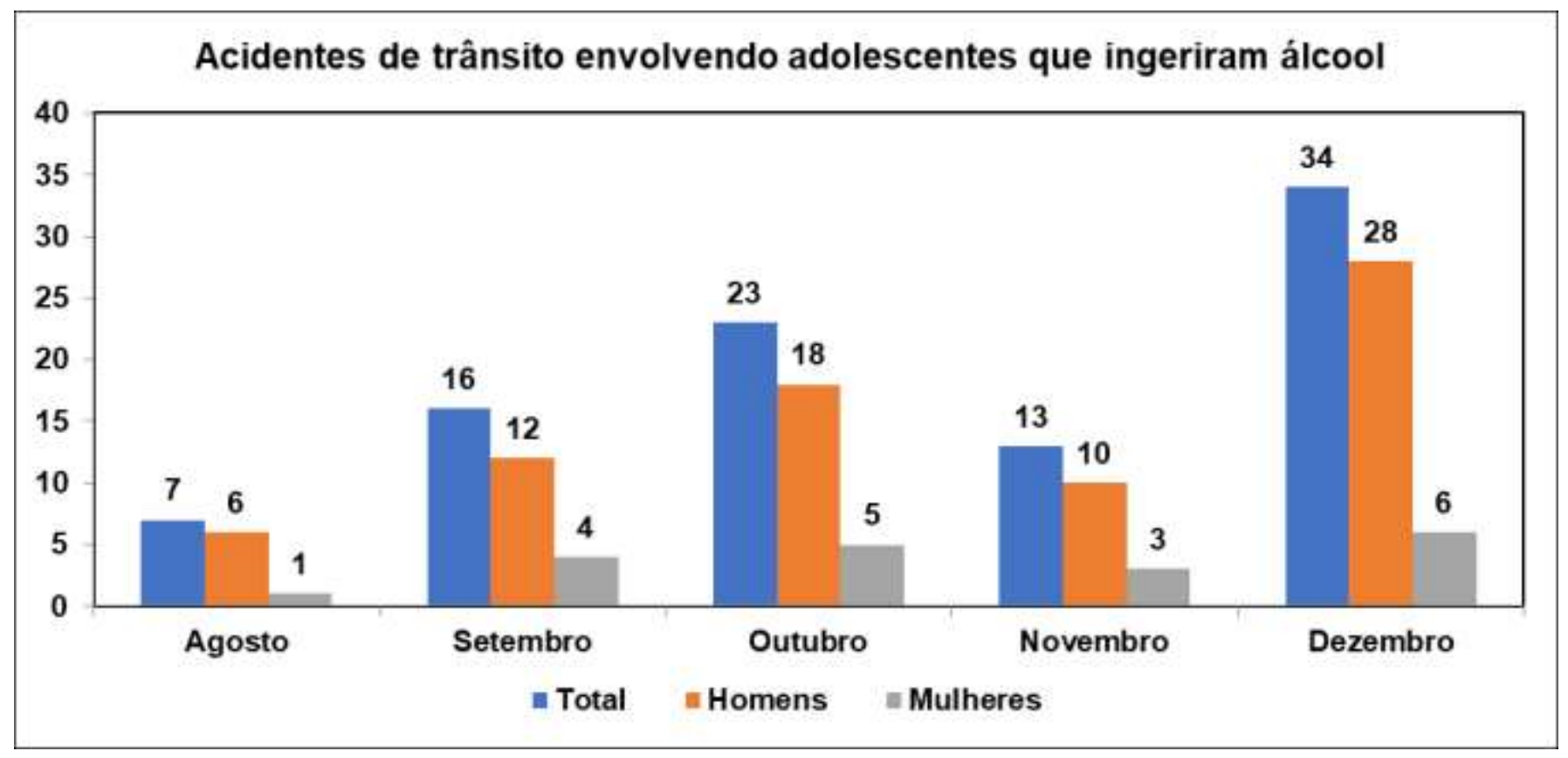

Fonte: Pesquisa de Campo (2020).

\section{Conclusão}

O presente estudo permitiu compreender a relação entre o consumo de bebidas alcoólicas por e acidentes de trânsito, caracterizando suas principais vítimas. Observou-se que as principais vítimas são os jovens e adolescentes, tendo aspectos como: inexperiência, a falta de habilitação e a imprudência, fatores condicionantes para o desenvolvimento dessa causa.

O estudo ponderou a obtenção de todos os fins propostos, pois, possibilitou identificar de forma mais abrangente todas as variáveis demonstradas estatisticamente nesta pesquisa, que ainda há muito que melhorar em relação aos fatores diretos ou indiretos pautados entre os pontos estudados, sendo eles, os adolescentes, acidentes de trânsito e a ingestão de bebidas alcoólicas, onde se faz necessário a utilização de medidas que viabilizem a construção e desenvolvimento de estratégias voltadas à melhoria da fiscalização diante das autoridades.

Por fim, faz-se necessário desenvolver medidas de conscientização e combate ao uso indevido de bebidas alcoólicas pelos adolescentes, e a elaboração de medidas de segurança pública mais eficazes para combater essa prática, além do desenvolvimento de estudos mais amplos e complexos, para que assim, seja possível a elaboração de políticas de educação em saúde pública, que tenha como objetivo principal, minimizar o desenvolvimento dessa prática, para obter, uma melhor perspectiva de vida e o bem comum em presença de todos.

\section{Referências}

Andrade, S. S. C. A., \& Jorge, M. H. P. M. (2016). Estimativa de sequelas físicas em vítimas de acidentes de trânsito internados no Sistema Único de Saúde. Rev. bras. epidemiol., 19(1), 100-111.

Barros, A. J. S., \& Lehfeld, N. A. S. (2007). Fundamentos de metodologia científica. Pearson Prentice Hall.

Benincasa, M., Tavares, A. L., Barbosa, V. M. M., Barbosa, M. P., Rezende, M. M., Heleno, M. G. V., \& Custódio, E. M. (2018). A influência das relações e o uso de álcool por adolescentes. Rev. Eletrônica Saúde Mental Álcool Drog., 14(1), 5-11.

Lei $n^{o}$ 9.503, 23 de setembro de 1997. Institui o Código de Trânsito Brasileiro. http://www.planalto.gov.br/ccivil_03/leis/19 503co mpilado.htm\#: :text=LEI\%20 N\%C2\%BA\%209.503\%2C\%20DE\%2023\%20DE\%20SETEMBRO\%20DE\%201997\&text=Institui\% 20o\%20C\%C3\% B3dig o\%20de\%20Tr\%C3\%A2nsito\%20Brasileiro.\&text=Art.\%201\%C2\%BA\%20O\%20tr\%C3\%A2nsito\%20de,rege\%2Dse\%20por\%20este\%20C\%C3\%B3digo. 
Research, Society and Development, v. 10, n. 8, e54410817731, 2021

(CC BY 4.0) | ISSN 2525-3409 | DOI: http://dx.doi.org/10.33448/rsd-v10i8.17731

Portaria $\mathrm{n}^{\circ}$ 466, de 12 de dezembro de 2012. Diretrizes e Normas Regulamentadoras de Pesquisa Envolvendo Seres Humanos. https://conselho.saude.gov.br/resolucoes/2012/Reso466.pdf.

Davoren, M. P., Demant, J., Shiely, F., \& Perry, I. J. (2016). Alcohol Consumption among University Students in Ireland and the United Kingdom from 2002 to 2014: A Systematic Review. BMC Public Health, 16(173), 1-13.

Dyniewicz, A. M. Metodologia da Pesquisa em Saúde para Iniciantes. (2009). Difusão Editora.

Ferreira, L. F. O., Veloso, L. U. P., Coutinho, N. F. L., Jr., Lira, V. F., \& Lira, J. A. C. (2019). Perception of adolescents about alcohol consumption. Rev Enferm UFPI, 8(2), 18-24.

Flick, U. Introdução à Metodologia de Pesquisa: um guia para iniciantes. (2013). Penso.

Lopes, G. T., Belchior, P. C., Felipe, I. C. V., Bernardes, M. M., Casanova, E. G., \& Pinheiro, A. P. L. (2012). Dinâmicas de criatividade e sensibilidade na abordagem de álcool e fumo com adolescentes. Revista de Enfermagem, 20(1), 33-8.

Melo, W. A., Oliveira, R. R., Brischiliari, A., Pelloso, S. M., \& Carvalho, M. D. B. (2018). Mortality trend due to traffic accident in young in the south of Brazil. Cad. saúde colet., 26(4), 360-368.

Moise, I. K. Geographic gender differences in traumatic unintentional injury hospitalization and youth drinking. Drug and Alcohol Dependence, 205, 1-35, 2019.

Oliveira, J. B., Kerr-Corrêa, F., Lopes, I. C., Vitti, W, Jr., Nunes, H. B. C., \& Lima, M. C. P. (2020). Alcohol use and risk of vehicle accidents: cross-sectional study in the city of São Paulo, Brazil. São Paulo Med J., 138(3), 208-215.

Ponce, J. C., Andreuccetti, G., Gonçalves, R. E. M., Gjerde, H., Bogstrand, S. T., Valen, A., \& Carvalho, H. B. (2019). Comparison of traffic data and blood alcohol concentration among fatally injured drivers in Norway and Sao Paulo, Brazil, 2005-2015. Traffic Injury Prevention, 20(7), 673-678.

Potter, P., \& Perry, A. G. Fundamentos de Enfermagem. (2013). Elsevier.

Robison, T., Tarzi, C., Zhou, X. G., \& Bailey, K. (2020). Screening for alcohol and substance use in pediatric trauma patients: A retrospective review. $J$ Pediatr Surg, 55(5), 921-925.

Rocha, M. B., Macacare, O. T., Abrahao, K. P., \& Ekuni, R. (2019). "Se beber, não dirija": popularizando os efeitos do álcool em um evento interativo. Interfaces - Revista de Extensão da UFMG, 7(1), 143-150.

Santos, R. O. M. (2014). Perfil epidemiológico dos acidentes de trânsito na cidade de Aracaju (Pós-graduação). Programa de Pós-graduação em Enfermagem, Centro de Ciências da Saúde, Universidade Federal de Santa Catarina, Florianópolis, SC, Brasil.

Smailovic, E., Lipovac, K., Pešić, D., \& Antić, B. (2019). Factors associated with driving under the influence of alcohol. Traffic Inj Prev, 20(4), $343-347$.

Soares, R. A. S., Pereira, A. P. J. T., Moraes, R. M., \& Vianna, R. P. T. (2012). Caracterização das vítimas de acidentes de trânsito atendidas pelo Serviço de Atendimento Móvel de Urgência (SAMU) no Município de João Pessoa, Estado da Paraíba, Brasil, em 2010. Revista Epidemiológica Serviço Saúde, 21(4), 589-600. 